\title{
Human Gut Microbial Taxa Metabolizing Dietary Obesogens: A BPA 1 directed-culturing and Bioinformatics Combined Approach
}

\section{Ana López Moreno}

University of Granada: Universidad de Granada https://orcid.org/0000-0003-3717-9852

\section{Ángel Ruiz-Moreno}

University of Granada: Universidad de Granada

Jesús Pardo

University of Granada: Universidad de Granada

Klara Cerk

University of Granada: Universidad de Granada

\section{Alfonso Torres-Sánchez}

University of Granada: Universidad de Granada

\section{Pilar Ortíz}

University of Granada: Universidad de Granada

\section{Marina Úbeda}

University of Granada: Universidad de Granada

\section{Margarita Aguilera ( $D$ maguiler@ugr.es )}

Department of Microbiology, Faculty of Pharmacy, University of Granada, Campus of Cartuja, 6 Granada 18071 Spain. https://orcid.org/0000-0002-3204-9787

\section{Research}

Keywords: culturomics, directed-culturing, obesogens, endocrine disruptors (ED), BPA, next-48 generation probiotics (NGP)

Posted Date: August 3rd, 2021

DOI: https://doi.org/10.21203/rs.3.rs-754318/v1

License: (c) (1) This work is licensed under a Creative Commons Attribution 4.0 International License.

Read Full License 


\section{Human gut microbial taxa metabolizing dietary obesogens: A BPA}

\section{directed-culturing and bioinformatics combined approach}

3 Ana López-Moreno ${ }^{1,2,3 \ddagger^{*}}$, Ángel Ruiz-Moreno ${ }^{1,2 \ddagger}$, Jesús Pardo ${ }^{1}$, Klara Cerk ${ }^{1,2}$, Alfonso Torres-Sánchez ${ }^{1,2}$,

4 Pilar Ortíz ${ }^{1,2}$, Marina Úbeda ${ }^{1}$ and Margarita Aguilera ${ }^{1,2,3^{*}}$

5 Author Affiliations:

61 Department of Microbiology, Faculty of Pharmacy, University of Granada, Campus of Cartuja,

$7 \quad$ Granada 18071 Spain.

82 Institute of Nutrition and Food Technology "José Mataix", Center of Biomedical Research, University

9 of Granada, 18016 Armilla, Granada, Spain.

103 IBS: Instituto de Investigación Biosanitaria ibs. 18012, Granada, Spain.

11 Author e-mail Addresses:

12 Ana López-Moreno: alopezm@ugr.es

13 Ángel Ruiz-Moreno: angel_trm_@hotmail.com

14 Jesús Pardo: jesusparugr99@gmail.com

15 Klara Cerk: klara.cerk@gmail.com

16 Alfonso Torres-Sánchez: alfons_ats@hotmail.com

17 Pilar Ortíz: piortiz@ugr.es

18 Marina Úbeda: marinaubeda@correo.ugr.es

19 Margarita Aguilera: maguiler@ugr.es

20 *Corresponding Authors: alopezm@ugr.es, maguiler@ugr.es Tel.: +34-9-5824-5129 (MA) 


\section{Abstract}

22 Background: Integrated data from culturomics and functional omics may depict holistic 23 understanding on gut microbiome eubiosis or dysbiosis, and microbial isolates can become a

24 source of differential enzymes and useful bioactive compounds. Culturing methods developed during last decade swift increases the importance of gut microbial isolates, focusing on media, modifications and conditions that propitiate cultured taxa that previously were considered fastidious or unculturable. In this context and focusing on gut microbiota dysbiosis triggered by obesogens and microbiota disrupting chemicals (MDC), we have conducted a directedculturing and bioinformatics combined approach, adding bisphenol A (BPA) and specific treatments to find resistant spore-forming bacteria, to obtain isolated strains for further explore their molecular BPA metabolizing or neutralizing capacities.

32 Results: Overall microbiota culturing media and conditions have been retrieved and organized 33 according to main gut taxa isolated during last decade. Furthermore, a catalogue of BPA 34 directed-cultured microorganisms has been obtained from 46 fecal samples from two 35 populations, children with obesity and normo-weight. A total of 235 BPA tolerating and 36 potentially BPA biodegrading microorganisms were mainly grouped to strictly anaerobic sporuled/non-sporuled, anaerobic facultative sporuled/non-sporuled. Firmicutes,

38 Enterobacteria and Actinobacteria species showed the major representation in both groups.

39 However, differential BPA tolerant microbiota composition from the populations was detected.

40 Bioinformatics analysis disclosed and predicted the variability of harboring genes encoding 41 specific enzyme for BPA biodegradation pathways that corroborated from directed-culturing 42 microbiota consortia obtained.

43 Conclusions: Strains from Staphylococcus, Bacillus and Enterococcus genera represented the 44 majority of the successfully cultured bacteria in both population specimens. From them, the 45 bioinformatics prediction assigned to Bacillus spp. the higher potential for BPA biodegradation. 
46 Therefore, extensive directed-culturomics approaches could be designed for different MDC

47 with common biodegradation pathways, such as parabens, phthalates, and benzophenones.

48 Keywords: culturomics, directed-culturing, obesogens, endocrine disruptors (ED), BPA, next49 generation probiotics (NGP).

\section{Background}

\section{Microbiota dysbiosis in obesity-related disorders triggered by exposure to ED and obesogens}

52 Currently, the exposure to obesogens and ED can lead to a microbial dysbiosis [1,2]. The

53 dysbiosis are based on misbalanced taxa compositions and associated to several metabolic 54 diseases, such as type 2 diabetes, obesity, and other endocrine disorders [3, 4, 5]. To isolate, 55 culture and analyze the microbial taxa components that can lead towards altered functional 56 effects would allow a better understanding of the pathophysiological mechanisms and its 57 prevention through the administration of beneficial microbes, helping to regulate the physiological hormonal axis [6]. Directed-culturing of microorganisms from obese and nonobese microbiota may lead to identify potential metabolizing and detoxifying strains, which could be used as NGP $[7,8]$.

61 The importance of culturomics for the human microbiome description is advancing towards more effective isolations via sophisticated culture methods of the human microbiome [9]. This method relies on intensively culturing human samples with different growth media under

64 different conditions, along with identifying any isolated bacterial colonies with matrix-assisted

65 laser desorption/ionization time of flight mass spectrometry (MALDI-TOF MS) and 16S rRNA

66 gene sequencing $[9,10]$. It showed its success in the isolation, description and characterization

67 of new bacterial species from the human microbiota $[9,11,12]$. This enabled the expansion of 68 the current human microbial database by reporting the isolation of a significant number of 
novel bacterial species and rendered the identification of previously considered "unclassified organisms" possible in clinical settings [12].

\section{Microorganisms detoxifying dietary obesogens: ED-Bisphenols}

72 EDs are considered as MDC [13]. Concretely, BPA is used in polycarbonate and epoxy resins

73 and packages. Its cumulative contamination reaches all kinds of environments, such as soils,

74 sediments, and aquatic environments, water, air and dust particles [14]. Several routes of human exposure to BPA have been described, including the digestive system (ingestion) through exposure to food packaging, drinking containers, dental monomers [15, 16]; the vertical transmission (maternofetal) [17]; the respiratory system (inhalation) [18]; and the integumentary system (skin and eye contact) though the thermal paper of the receipts, eyeglass lenses and feminine hygiene products $[19,20]$. The presence of this obesogen or MDC in humans has been confirmed by detecting it in human serum, urine, saliva, hair, tissue and blood [21, 22]. Thus, BPA removal from the natural environment is an increasing worldwide concern and several

82 studies identified biological effective via to remove BPA from the environment through 83 organisms such as bacteria, fungi, algae and plants [23, 24]. However, there are still no clear 84 clinical studies aimed at eliminating or reducing the amount of exposure to BPA in the human body. The demonstrated evidence of the effects of BPA as an ED and its transfer to foods has led the industry to use analogous compounds such as bisphenol S (BPS). However, recently studies have shown that some of these analogues may be even more harmful than BPA [25]. In this case, BPS has also been shown to act as an ED but investigation in this field has remained limited [26]. Moreover, the use of NGP is increasing due to the specific knowledge of the

90 human intestinal microbiota and the possibility of intervening and modulating the dysbiosis 91 determined by certain diseases. Culturomics remains as main strategy for the isolation of new 92 gut microorganisms. 
93 The BPA-degradation capabilities from some microorganisms, like Bacillus spp., have been

94 studied as an environmental and bioremediation resource [27, 28]. Furthermore, species from

95 this genus have been isolated from infant fecal samples with the four complete molecular

96 pathways of BPA degradation [29]. However, while the use of BPA-degrading microorganisms

97 is widely extended in bioremediation, based on a previous review [30] there were no clinical

98 trials involving beneficial microorganisms, metabolic diseases and xenobiotic obesogens. This

99 fact may indicate a new area of research where NGP with the ability to modulate the microbiota

100 are used, counteracting the impact of xenobiotics ingested through the diet.

101 This work focuses on promoting the knowledge regarding culturomics data searching and

102 directed culturing through different microbial culture techniques to increase the catalogue of 103 isolated microorganisms from human gut microbiota, more specifically, the approach focus on

104 the BPA tolerant and/or biodegrader bacteria.

\section{Material and Methods}

\section{Culturomics review data for increasing the microbiota taxa isolates}

107 Literature search and review of studies were developed in collaboration with Granada

108 librarian support using medical subject headings (MeSH) and the key words (see below) under

109 a stepwise procedure search and adapted to each database's tutorials. The following electronic

110 databases were searched from October 2020 to July 2021: PubMed, Web of Science (Thomson

111 Reuters Scientific) and Scopus (Elsevier). The reviewers revised titles and abstracts, then full-

112 text publications with reference to the inclusion criteria that were all the studies about

113 culturomics or culturing from human gut microbiota, the key word were (Culturomics* AND

114 microbiota), Culturing* AND microbiota AND obesity AND "endocrine disrupt";

115 Culturomics* and microbiota and obesity and xenobiotic*; Culturing * and microbiota and

116 obesity and hormon*; Culturing * and microbiota and obesity and "drug metabol*"; Culturing * 
117 and microbiota and "metabolic syndrome" "endocrine disrupt"; Culturomics * and microbiota

118 and "metabolic syndrome" and xenobiotic*; Culturomics * and microbiota and "metabolic

119 syndrome" and hormon*; Culturomics * and microbiota and "metabolic syndrome" and "drug

120 metabol"; ${ }^{* \prime}$ Culturomics * and microbiota and diabetes and "endocrine disrupt" ${ }^{* \prime}$; Culturomics *

121 and microbiota and diabetes and xenobiotic ${ }^{*}$; Culturomics ${ }^{*}$ and microbiota and diabetes and

122 hormon*; Culturomics * and microbiota and diabetes and "drug metabol"; Culturomics * and

123 microbiota and fertility.

124 Experimental Culturomics approach to isolate gut microbes metabolizing obesogenic ED

125 BPA Directed-Culturing approach for the isolation of microbiota strain catalogue

126 A common approach to isolate microbial strains from microbiota has been pursued in our 127 research team [29]. For this study, 235 microbial isolates from fecal human microbiota 128 collections of 6-12 years-old children (Isolates-Project OBEMIRISK) appropriately maintained at $129-80{ }^{\circ} \mathrm{C}$ underwent a directed culturing approach adding BPA to searching tolerant and

130 potentially BPA biodegrading microorganism by a serial dilution method, and exposition to

131 different BPA concentrations [0.5, 10, 20, and $50 \mathrm{ppm}]$ during $72 \mathrm{~h}$ at $37^{\circ} \mathrm{C}$ and further spreading

132 in different media and incubated under aerobic and under anaerobic cultivation performed

133 with Anaerocult ${ }^{\circledR}$ A system (Merck, Darmstadt, Germany) at $72 \mathrm{~h}$ and $37{ }^{\circ} \mathrm{C}$. Different 134 conditions and culture mediums were used for optimizing the uncultured bacterial growth 135 including Brain Heart Infusion (BHI), Man, Rogosa and Sharpe (MRS), Reinforced Clostridial

136 Medium (RCM), Gifu Anaerobic modified Medium (GAMm) agar/gellan [31]. Isolated BPA-

137 tolerant bacterial colonies with distinguishing features were isolated as pure culture for

138 subsequent morphological, phenotypic and genotypic identifications: bacterial cell counts, gram

139 staining, spore staining, capsule staining, catalase activity, oxidase, and motility tests. 
140 BPA Directed-Culturing and Spore-forming searching taxa components: Clostridium spp.

141 and Bacillus spp.

142 In parallel, a specific treatment was carried out to favor the isolation of spore-forming bacteria.

143 For this, after the exposure to BPA and before the spread on the media, the samples were

144 homogenized in $70 \%$ ethanol for $4 \mathrm{~h}$ and treated with a bile acids solution $(0,1 \mathrm{mg} / \mathrm{ml}$ of bile

145 bovine in PBS) for the metabolic activation of the spores. Then, the samples were processed and

146 analyzed as described above. The 16s rRNA from all the isolated colonies were analyzed.

\section{Genomic DNA extraction, Taxonomy Identification and Phylogenetic Analysis}

148 Genomic DNA was extracted using DNAeasy columns (Qiagen ${ }^{\circledR}$, Germany) following the

149 manufacturing instructions. The isolated DNA was quantified using Nanodrop (Thermo

150 Scientific) and biophotometer (Eppendorf® D30). The quality of DNA was monitored through

151 gel electrophoreses. Complete 16S RNA gene sequencing of selected bacterial strains was done

152 by Sanger method (Institute of Parasitology and Biomedicine "López-Neyra" (IPBLN) Service).

153 Forward and reverse sequences were provided separately. Reverse sequence was converted to

154 complementary sequence with Chromas Pro 2.0 software (Technelysium Pty Ltd., Tewantin,

155 Australia). Sequences were examined for maximum homology against GenBank using National

156 Center of Biotechnology Information's (NCBI) BLASTn program. The collection and

157 phylogenetic comparison of 16S RNA partial gene sequences was done using the Ezbiocloud

158 platform [32].

159 Genome data mining tools for prediction of BPA metabolic maps and enzymatic pathways in

160 whole genome sequencing (WGS) Type strains from the closest isolated species and isolated

161 from microbiota

162 In order to discover the presence of BPA biodegradation gene potential of cultured microbiota,

163 several bioinformatics tools were used to perform genome mining. A data retrieving program 
164 has been specifically computed using Pascal programming language to obtain the BPA

165 pathways enzymes ID and the corresponding Loci from the microbial genomes.

166 Type strains genomes from the closest species isolated were retrieved from NCBI Genome Data

167 Bank in GenBank file format in order to list the proteins that they were able to potentially

168 encode the enzymes.

169 A more detailed prediction of the clusters was performed by checking the downstream and

170 upstream genes of those involved in BPA biodegradation using NCBI genome map viewer.

171 The identification of BPA genes encoding enzymes involved on the four biodegradation

172 pathways was carried out by the analysis of the WGST of type strains, following the same

173 approach explained above.

\section{Results and Discussion}

\section{Microbiota culturing approaches, media and conditions for isolation of gut microbial taxa}

176 Theoretical searching on culturomics data, which were thoroughly analyzed, allowed retrieving

177 main culturing media and conditions used for isolation of relevant gut microbiota taxa

178 components are summarized in Table 1. This data extraction analysis displays at once a batery

179 of media for susscessful isolating of specific species belonging to genera from phyla Firmicutes,

180 Bacteroidetes, Actinobacteria, and alpha-Proteobacteria and information on their oxygen

181 tolerance: aerobic, aerotolerant anaerobe; strictly anaerobic; and facultative anaerobe. Main

182 media retrieved were: BCB (Blood Culture Bottle), BHI, BRU (Brucella medium), CBA

183 (Columbia Blood Agar), CHRIS (Christensenella medium), CNA (Columbia NaladixicAcid

184 Agar), COS (Columbia agar liquid medium $+5 \%$ sheep blood), CPVX (Chocolate agar +

185 PolyViteX), GAM (Gifu Anaerobic Media), MB (Marine Broth), MRS (Man, Rogosa and Sharpe),

186 RM (R-Medium), RCA (Reinforced Clostridial Agar), SCM (Schaedler Medium), TSB

187 (Trypticase Soy Broth), YCFA (Yeast extract-casein hydrolysate-fatty acids), WC (Wilkins 
188 Chalgren) with several modifications with supplements such as vitamins, blood, rumen fluid,

189 biliary salts, ethanol and several conditions collected in additional files (Supplementary

190 Material Excel Table 1S; Excel Table 2S).

191 Similarly, useful information on favoured cultured isolates from gut microbiota acting as

192 beneficial microorganisms or potential NGP was previously retrieved. Main media and

193 pertinent modifications for isolating obesity and anti-obesity probiotics were: BHI, GAM, Gut

194 Microbiota Medium (GMM), Lactobacillus selection (LB), MRS, YCFA, and BPA-added media

195 [8]. Therefore, culturomics efforts contributed to enlarge the repertoire of isolated bacterial

196 species from humans by $28 \%$ and provided biological material to the scientific community that

197 can be further studied for its role and interaction with other bacterial species and host [33].

198 Conversely, the efficient molecular methods, such as metagenomics, which aims to describe the

199 human microbiota with no culture efforts, needed complementary developing fields. However,

200 some drawbacks are encountered that require the use and development of comparing culture

201 approaches [34], such as sequencing depth bias [34, 35], incomplete genomic databases [12, 33,

20236 ] or the ability to distinguish between live and dead bacterial DNA in the studied samples

203 [36]. In a recent study that examined the gut microbiota composition of 8 healthy individuals, it

204 was shown that culturomics enabled $20 \%$ higher bacterial richness in comparison to

205 metagenomics [37]. Interestingly, isolated species' genome sequences enlarged by $22 \%$ the data

206 obtained by metagenomics analyses and showed that the number of species recovered by

207 culture is higher than the number of species detected by metagenomics [37]. 
Table 1. Culturing media and conditions for isolation microbiota taxa components (Aer: Aerobic; Aan: Aerotolerant Anaerobe; SAn: Strictly Anaerobic; FAn: Facultative Anaerobe)

Species / Oxygen Tolerance

Bacillus spp. / Aer / AAn /FAn

$[9,38,39,40,41]$

Blautia spp. / SAn

[38, 39, 41, 42, 43, 44, 45]

Clostridium spp. / SAn

$[11,40,41,42,46,47,48]$

Dialister spp. / SAn [41]

Enterococcus spp. / FAn [40, 41, 49]

Eubacterium spp. / SAn [40, 41]

Lactobacillus spp. / AAn [41]

Megasphaera spp. / SAn [41, 50]

Peptoniphilus spp. / San $[9,11,41]$

Ruminococcus spp. / SAn [9, 11, 41, 42]

Staphylococcus spp. / FAn [40, 41]

Streptococcus spp. / FAn [40, 41]

Alistipes spp. / SAn [9, 11, 40, 41, 42

51]

Bacteroides spp. / SAn [11, 40, 41]

Butyricimonas spp. / SAn [11, 40, 41]

Parabacteroides spp. / SAn / [40, 41]

Prevotella spp. / SAn [40, 41, 42, 52] Actinomyces spp. AAn $[9,11,42]$

Bifidobacterium spp. / An/SAn [40, 41]

Collinsella spp. / SAn [11, 40, 41]

Corynebacterium spp. /AAn $[11,41,51]$

Propionibacterium spp. / AAn [41]

$\frac{1}{\delta}$
BCB38; BCB02; BCB03; ВCB04; BCB05; BCB06; BCB08; BCB09; BCB10; COS01; COS03; MB01; MB02; TSB01; BHI01; BCB19; YCFA06; BCB23; COS09; TSB04; BCB07; YCFA02; MB03; TSB03; BCB18; ВCB01; COS02; COS04; BHI02; CBA01; MRS02; BCB37; ВCB33; BHI07; BCB36; BCB46; ВCB14; BCB15; BCB12; BBCB22; BHI04; BCB13; YCFA01; MB04; BCB55

BHI05; ВCB13; ВCB15; ВСB01; ВСВ03; ВCB05; ВСB07; ВCB09; ВCB10; COS02; MB02; TSB04; YCFA05; CBA01; BCB52; RM01; BCB11; CNA01; YCFA01; BCB28; BCB19; COS09; YCFA03; WC02; CBA02; GAM02; RCA02

ВСB01; ВСВ03; ВСВ05; ВСB07; ВСB09; ВCB10; COS04; MB02; ВCB15; COS02; RM01; RCA01; BCB34; BCB39; CHRIS01; CBA01; BCB19; COS09; YCFA05; BCB13; TSB04; CBA02; YCFA01; SCM04; YCFA04; MB04; RM02; BCB49; ВCB28; BCB25; CNA01; BCB17; BCB21; BCB50; BCB32; BCB02; BCB04; BCB11; WC01; BHI02; YCFA03; RCA02; WC02; BHI03; BCB33; BCB30; TSB02; BCB31; YCFA02; MRS02; RM03; COS03; TSB01; BCB22; COS08; MB03; TSB03; BCB06; MRS01; BHI01; BCB23; BCB12; BCB14; BCB16; BCB20

BCB07; CHRIS01; SCM04; RM01; RM02; BCB11; BCB19; COS02; ВCB01; ВCB03; BCB05; BCB09; BCB10; COS04; MB02; YCFA01; MRS01

CBA03; YCFA04; YCFA06; BCB04; BCB07; BCB06; BCB08; COS01; COS03; COS04; MB01; MB02; TSB01; YCFA01; CHRIS01; MRS01; SCM04; RM01; BCB23; BCB11; BCB17; BCB22; BCB19; BCB20; COS09; MB03; TSB03; TSB04; BCB10; RM02; BCB01; BCB03; COS02; BCB05; BCB09; BHI01; BCB02; CNA01; RM03; SCM01; YCFA02; RCA01; BCB15; BCB21; TSB02; WC01; BHI02; CBA01; MRS02; BCB13; BCB14; COS08; MB04; BCB12; BCB16; YCFA03; RCA02; WC02; BHI03; MRS03

BCB07; SCM04; BCB15; MB02; BCB19; BCB01; BCB05; BCB09; COS02; COS04; RM01; RCA01; YCFA04; BCB03; BCB11; WC01; CBA01; COS09; BCB13; MB04

ВCB07; COS04; SCM04; CNA01; BCB10; COS02; YCFA01; MRS01; RM01; BCB11; YCFA02; CHRIS01; BCB15; BCB19; COS09; BHI03; BCB02; BCB03; BCB04; BCB06; COS01; COS03; MB01; MB02; TSB01; BHI01; BCB13; RCA01; RM02; BCB23; RM03; SCM01; CBA01; MRS02; BCB01; BCB09; BCB05; WC01; BHI02

COS02; COS04; RM01; BCB07; YCFA01; BCB09; BCB10; SCM04; BCB31

CHRIS01; BCB01; BCB05; BCB07; MB02; BCB10; COS02; COS04; RM02; BCB35; YCFA01; MRS01; RM01; BCB53; BCB15; BCB03; BCB09; SCM04; BCB11; BCB38; BCB40; YCFA03

YCFA05; BCB11; RM03; SCM01; YCFA02; CBA01; BCB13; BCB15; BCB19; BCB03; BCB07; BCB09; COS02; RCA02; BHI03; BCB40; BCB41; RM01; RM02; TSB04; YCFA01; CHRIS01; SCM04; CNA01; BCB05

YCFA06; ВCB01; ВCB02; ВCB03; ВCB07; ВCB06, BCB10; COS01; COS04; MB01; MB02; YCFA01; RM01; RM02; BCB11; BCB15; BCB19; COS09; MB04; BCB05; BCB14; BCB17; BCB20; COS08; MB03; TSB03; BCB08; BCB09; CHRIS01; BCB04; COS02; COS03; BHI01; CBA01

BCB07; YCFA04; BCB04; BCB05; BCB10; MB02; RM01; CBA01; BCB06; COS02; BHI01: YCFA01; BCB02; BCB09; COS01; COS03; BCB03; COS04; BCB23; CNA01; BCB01; CHRIS01; SCM04; BHI02; BCB08; TSB01; MRS01; RCA01; WC01; YCFA06; BCB11

YCFA05; ВCB01; ВCB03; BCB05; BCB07; BCB09; BCB10; COS02; COS04; RM02; BCB11; CNA01; YCFA02; WC01; CBA01; BCB19; YCFA01; CHRIS01; SCM04; BCB48; MB02; BHI02; CPVX01; BCB13; BCB24; MRS01; TSB04; YCFA04; RCA01; BRU02; SCM01; BCB15; COS09; MB04; SCM02; RM03; BCB27

ВСВ01; ВСВ03; ВСВ05; ВСB07; ВСB09; ВCB10; COS02; COS04; МB02; ВCB11; SCM01; YCFA02; RCA01; WC01; BHI02; CBA01; MRS02; BCB19; RM03; BCB13; CBA02; SCM04; YCFA04; CNA01; RM01; TSB04; BCB15; RM02; YCFA01; CHRIS01; MB04; TSB03; WC02; COS09; YCFA03; TSB02

BCB41; ВCB01; ВCB03; ВCB05; ВCB07; BCB09; BCB10; COS02; MB02; CBA01; YCFA04; CHRIS01; SCM04; RM02; BCB11; SCM01; COS09; YCFA02; CNA01; BCB19

BCB05; BCB07; COS02; COS04; SCM04; RM02; CBA01; BCB19; YCFA04; CHRIS01; RM01; BCB11; CNA01; SCM01; BCB15; TSB04; BCB01; BCB03; BCB09; BCB10; BHI01; WC01; BHI02; YCFA01; MB02; YCFA02; RCA01

BCB10; COS02; RM01; BCB05; BCB01; BCB07; BCB09; YCFA01; CHRIS01; WC01; BCB11; CNA01; CBA01; YCFA05; SCM01; SCM04; CBA04; BRU03; BCB19; BCB03

ВСB03; ВCB09; YCFA02; CBA01; COS09; BCB19; ВCB07; MB02; BCB48; BCB11; BCB42

BCB07; BCB10; YCFA01; MRS01; SCM04; RM01; RM02; BCB11; CNA01; RM03; SCM01; CBA01; BCB15; BCB19; COS09; BCB01; BCB03; BCB05; YCFA02; RCA01; WC01; BHI02; COS02; MB02; CHRIS01; MRS02; BCB13; BCB17; YCFA03; WC02; BHI03; CBA02; MRS03; RCA02; BCB09; COS04; YCFA04; BCB23

YCFA04; BCB05; BCB07; COS02; YCFA01; CHRIS01; RM01; BCB11; CNA01; RM03; SCM01; CBA01; BCB13; BCB15; BCB19; SCM04; RM02; BCB01; CBA02; MRS02; BCB41; MB07; BCB10; YCFA02; BCB23; COS09

SCM04; RM02; BCB11; BCB23; CPVX02; COS02; BCB44; BHI01; BCB07; COS04; BCB10; MRS01; CBA03

BCB07; YCFA01; RM02; BCB11; BCB19; MB04; TSB04; YCFA03; CHRIS01; MRS01; SCM04; RM01; BCB09; MRS02; BCB02; BCB06; BCB10; MB01; MB02

BHI08; COS04; MB02; RM01; RM02; YCFA04; MRS01; BCB23; YCFA06; BCB11; BCB02; BCB03; BCB04; BCB07; BCB09; COS01; COS02; MB01; BHI01 


\section{BPA Directed-Culturomics approach}

We identify 192 bacteria isolates from human gut microbiota with high BPA tolerance [>20 ppm]. They were isolated from general media, supplemented with BPA, without searching for associated taxa, in following order: BHI (80 isolates), MRS (49 isolates), RCM (30 isolates), GAMa (18 isolates) and GAMg (15 isolates) without any specific media for associated taxa. The overall mean values

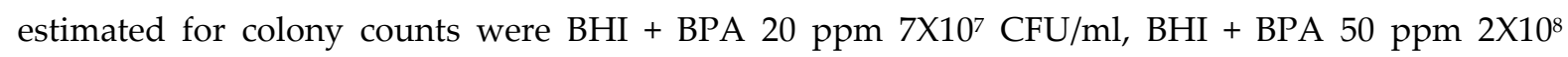

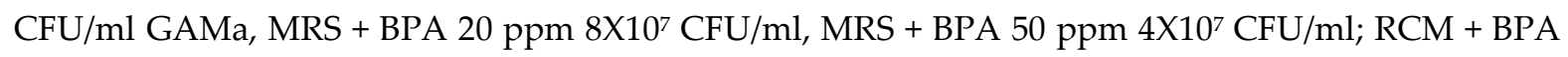

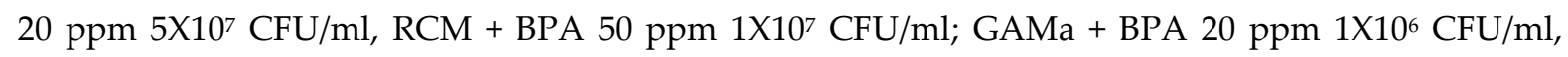
GAMa + BPA 50 ppm 5X105 CFU/ml; GAMa + BPA 20 ppm 5X10 6 CFU/ml, GAMa + BPA 50 ppm $2 \mathrm{X} 10^{6} \mathrm{CFU} / \mathrm{ml}$. It is interesting to highlight that taxa from Actinobacteria phylum with high BPA tolerance were isolated only in BHI medium. The relative abundance of these isolates, together with taxonomically closest species, maximum BPA concentration tolerated and specific media for isolation are detailed in Table 2 for normo-weight children specimens analyzed and Table 3 for specimens from children with obesity. A phylum grouping data analysis showed differences in relative abundance of cultured Firmicutes, Proteobacteria and Actinobacteria between both populations. Firmicutes were the most abundant phylum with BPA tolerance found, representing $72 \%$ in normoweight children and $73 \%$ in children with obesity. Proteobacteria was differentially represented in both groups by $17 \%$ and $20 \%$, respectively. However, dataset showed differences in Actinobacteria and uncultured bacteria groups, Actinobacteria group represented $6 \%$ of the bacteria isolated in normo-weight children and 5\% in children with obesity, in comparison to uncultured bacteria that represented $5 \%$ of the total bacteria isolated in normo-weight group, and $3 \%$ in population with obesity. Similarly, xenobiotics and specifically BPA tolerance by specific gut microorganisms was previously described for the traditional probiotics Bifidobacterium breve strain Yakult (BbY) and Lactobacillus casei strain Shirota (LcS) that showed protective effects against BPA dietary exposure in rats by reducing the intestinal absorption of BPA and facilitating its excretion [54]. Similarly, Lactococcus lactis strains adsorbed BPA but not degrade it [55]. Bioaccessible BPA decreased after 
digestion and this exposure changed microbial community, up-regulating the abundance of BPA-

degrading bacteria, such as Microbacterium and Alcaligenes [56].

Table 2. BPA tolerant cultured bacteria taxa from normo-weight microbiota.

\begin{tabular}{|c|c|c|c|c|}
\hline $\begin{array}{c}\text { Representative } \\
\text { Isolates (\%) }\end{array}$ & $\begin{array}{c}\text { Closest species } \\
\text { Accession Number }\end{array}$ & $\begin{array}{c}\text { Similarity of } \\
\text { partial 16S } \\
\text { rRNA }(\%)\end{array}$ & $\begin{array}{l}\text { BPA [ppm] } \\
\text { Treatments }\end{array}$ & Media \\
\hline \multicolumn{5}{|c|}{ Firmicutes } \\
\hline 11 & Bacillus velezensis |NC_009725.1| & 100 & 50 & $\mathrm{BHI} / \mathrm{MRS}$ \\
\hline 1 & Bacillus amyloliquefaciens IMW363310.1। & 100 & 100 & $\mathrm{BHI}$ \\
\hline 3 & Bacillus subtilis |HQ333016.1| & 99.54 & 50 & BHI/MRS \\
\hline 1 & Bacillus nealsonii |NR_044546.1| & 99.66 & 50 & GAMg \\
\hline 2 & Bacillus altitudinis | MT627439.1| & 100 & 50 & $\mathrm{BHI}$ \\
\hline 1 & Lacticaseibacillus paracasei ICP039707.1। & 99.74 & 10 & $\mathrm{RCM}$ \\
\hline 2 & Lysinibacillus fusiformis ICP026120.1। & 100 & 50 & MRS \\
\hline 1 & Bifidobacterium animalis IMT613598.1| & 98.65 & 20 & MRS \\
\hline 15 & Enterococcus faecium IMW816627.1। & 100 & 50 & $\mathrm{BHI} / \mathrm{MRS} / \mathrm{GAMg} / \mathrm{RCM}$ \\
\hline 6 & Enterococcus faecalis INR_113902.1| & 100 & 20 & $\mathrm{BHI}$ \\
\hline 1 & Enterococcus durans |MT545097.1| & 99.89 & 50 & GAMg \\
\hline 1 & Enterococcus mundtii |AP019810.1| & 99.83 & 20 & GAMg \\
\hline 1 & Enterococcus lactis |MZ475096.1| & 100 & 20 & $\mathrm{RCM}$ \\
\hline 3 & Enterococcus hirae |KX752868.1| & 99.85 & 50 & $\mathrm{RCM}$ \\
\hline 1 & Staphylococcus capitis |CP053957.1| & 100 & 20 & $\mathrm{BHI}$ \\
\hline 2 & Staphylococcus caprae INR_119252.1| & 99.39 & 50 & MRS \\
\hline 2 & Staphylococcus cohnii |MK465351.1| & 99.88 & 50 & BHI/GAMa \\
\hline 3 & Staphylococcus epidermidis |CP040883.1| & 100 & 50 & $\mathrm{RCM}$ \\
\hline 1 & Staphylococcus saprophyticus ICP054831.1| & 100 & 50 & $\mathrm{BHI}$ \\
\hline 1 & Turicibacter sanguinis |CP053187.1| & 91.26 & 50 & $\mathrm{BHI}$ \\
\hline 1 & Clostridium tertium |JX267105.1| & 99.59 & 20 & $\mathrm{BHI}$ \\
\hline 1 & Clostridium symbiosis |KR364763.1| & 99.49 & 20 & $\mathrm{BHI}$ \\
\hline 6 & Clostridium paraputrificum INR_113021.1| & 99.65 & 50 & BHI/GAMa \\
\hline \multirow[t]{2}{*}{1} & Paraclostridium bifermentans IMT604800.1| & 100 & 50 & RCM \\
\hline & Proteobacteria & & & \\
\hline 9 & Escherichia coli IMH511549.1| & 99.87 & $20 / 50$ & BHI/MRS/GAMa \\
\hline 1 & Escherichia coli |CP059988.1| & 93.09 & 50 & $\mathrm{BHI}$ \\
\hline 1 & Escherichia coli ICP046009.1| & 91.35 & 50 & $\mathrm{BHI}$ \\
\hline 1 & Escherichia fergusonii |NR_074902.1| & 99.79 & 20 & $\mathrm{BHI}$ \\
\hline 1 & Pseudomonas synxantha ICP074078.1। & $77.83^{*}$ & 20 & GAMg \\
\hline 1 & Pseudomonas parafulva | IMT367815.1 & 100 & 50 & GAMa \\
\hline 1 & Enterobacter hormaechei ICP027111.1। & 99.80 & 50 & $\mathrm{RCM}$ \\
\hline \multirow[t]{2}{*}{1} & Acinetobacter radioresistens IMT367790.1। & 99.76 & 20 & $\mathrm{RCM}$ \\
\hline & Actinobacteria & & & \\
\hline 1 & Rothia dentocariosa |CP054018.1| & 99.86 & 100 & $\mathrm{BHI}$ \\
\hline 5 & Microbacterium paraoxydans INR_115540.1| & 98.76 & $20 / 50$ & $\mathrm{BHI}$ \\
\hline 5 & Uncultured bacteria & & $10 / 20 / 100$ & $\mathrm{RCM} / \mathrm{BHI}$ \\
\hline
\end{tabular}


Table 3. BPA tolerant cultured bacteria taxa from obese microbiota.

\begin{tabular}{|c|c|c|c|c|}
\hline $\begin{array}{l}\text { Representative } \\
\text { Isolates (\%) }\end{array}$ & $\begin{array}{l}\text { Closest species } \\
\text { Accession Number }\end{array}$ & $\begin{array}{c}\text { Similarity of } \\
\text { partial 16S } \\
\text { rRNA (\%) }\end{array}$ & $\begin{array}{l}\text { BPA [ppm] } \\
\text { Treatments }\end{array}$ & Media \\
\hline \multicolumn{5}{|c|}{ Firmicutes } \\
\hline 12 & Bacillus velezensis INR_075005.2| & 99.40 & $20 / 50 / 100$ & BHI/MRS/GAMa \\
\hline 1 & Bacillus amyloliquefaciens I MW363310.1 | & 100 & 20 & $\mathrm{RCM}$ \\
\hline 2 & Bacillus subtilis |MN393073.1| & 100 & 50 & GAMg \\
\hline 1 & Bacillus pacificus ICP041979.1| & 99.74 & 50 & GAMa \\
\hline 1 & Bacillus cereus |KX161843.1| & 99.11 & 20 & MRS \\
\hline 1 & Bacillus paramycoides |MT538529.1| & 99.89 & 20 & $\mathrm{RCM}$ \\
\hline 1 & Bacillus altitudinis | MT627439.1| & 99.84 & 20 & GAMg \\
\hline 1 & Bacillus circulans |MT294022.1| & 99.87 & 50 & MRS \\
\hline 2 & Bacillus safensis |MT377905.1| & 99.10 & 20 & $\mathrm{BHI}$ \\
\hline 1 & Bacillus licheniformis IMT642945.1| & 100 & 20 & MRS \\
\hline 2 & Lacticaseibacillus casei | KF673514.1| & 99.77 & 20 & MRS \\
\hline 4 & Latilactobacillus sakei |NR_113821.1| & 99.85 & 10 & MRS \\
\hline 1 & Lysinibacillus fusiformis IMT605500.1 | & 99.71 & 50 & $\mathrm{BHI}$ \\
\hline 18 & Enterococcus faecium |MN453594.1| & 97.95 & $20 / 50$ & BHI/MRS/GAMa \\
\hline 3 & Enterococcus faecalis IMT611694.1| & 99.73 & 20 & $\mathrm{BHI} / \mathrm{RCM}$ \\
\hline 4 & Enterococcus lactis IMZ475096.1| & 100 & 50 & $\mathrm{BHI}$ \\
\hline 3 & Enterococcus hirae INR_114743.1| & 99.85 & 20 & $\mathrm{BHI}$ \\
\hline 1 & Enterococcus gallinarum INR_104559.2 | & 99.77 & 50 & $\mathrm{BHI}$ \\
\hline 5 & Staphylococcus epidermidis ICP043804.1| & 99.87 & $20 / 50$ & BHI/RCM/GAMa \\
\hline 1 & Staphylococcus caprae |NR_119252.1| & 100 & 50 & $\mathrm{BHI}$ \\
\hline 1 & Staphylococcus capitis INR_027519.1| & 100 & 10 & MRS \\
\hline 1 & Staphylococcus saprophyticus INR_041324.1 | & 99.55 & 20 & BHI \\
\hline 1 & Turicibacter sanguinis |CP053187.1| & 100 & 50 & $\mathrm{BHI}$ \\
\hline 5 & Clostridium paraputrificum |MN055965.1| & 97.47 & 50 & GAMg \\
\hline 1 & Clostridium desporicum INR_026491.1 | & 99.46 & 20 & GAMg \\
\hline 1 & Clostridium tertium |MT539087.1 | & 100 & 50 & BHI \\
\hline \multicolumn{5}{|c|}{ Proteobacteria } \\
\hline 13 & Escherichia coli |CP053231.1| & 99.87 & $20 / 50$ & MRS/BHI \\
\hline 1 & Escherichia fergusonii |MT912775.1| & 99.25 & 10 & $\mathrm{BHI}$ \\
\hline 1 & Shigella flexneri |JX307691.1| & 99.87 & 50 & $\mathrm{RCM}$ \\
\hline 1 & Pseudomonas parafulva |MT367815.1| & 100 & 50 & MRS \\
\hline 1 & Enterobacter cancerogenus |MT557032.1| & 100 & 20 & $\mathrm{RCM}$ \\
\hline 1 & Raoultella ornithinolytica IMF462255.1। & 100 & 50 & $\mathrm{BHI}$ \\
\hline 2 & Acinetobacter radioresistens INR_114074.1| & 99.80 & 10 & BHI \\
\hline 1 & Burkholderia contaminans IHQ746879.1| & 99.18 & 20 & MRS \\
\hline \multicolumn{5}{|c|}{ Actinobacteria } \\
\hline 1 & Microbacterium oxydans |MT533951.1| & 100 & 50 & $\mathrm{BHI}$ \\
\hline 1 & Micrococcus luteus ICP043842.1। & 99.13 & 20 & $\mathrm{BHI}$ \\
\hline 1 & Kocuria rhizophila INR_027193.1I & 100 & 20 & $\mathrm{BHI}$ \\
\hline 3 & Uncultured bacteria & & $20 / 50$ & $\mathrm{BHI}$ \\
\hline
\end{tabular}

All the sequences were submitted to GenBank under the Accession Numbers: MZ614066-MZ614252. 
Most dominant BPA tolerant genera were Enterococcus sp., Bacillus sp., Escherichia sp., Staphylococcus sp. in both populations (Fig. 1) representing near $75 \%$ of the taxa found. However, we can see differences between both groups in the minority BPA tolerant genera, some of these genera are exclusive of each population, conforming differential microbiota composition according to normo-weight children or children with obesity. The minority BPA tolerant genera found exclusively in normo-weight children were Rothia sp., Paraclostridium sp. and Bifidobacterium sp. However, Kocuria sp., Micrococcus sp., Burholderia sp., Raoultella sp., Shigella sp., and Latilactobacillus sp. were found exclusively in overweight and obese children.

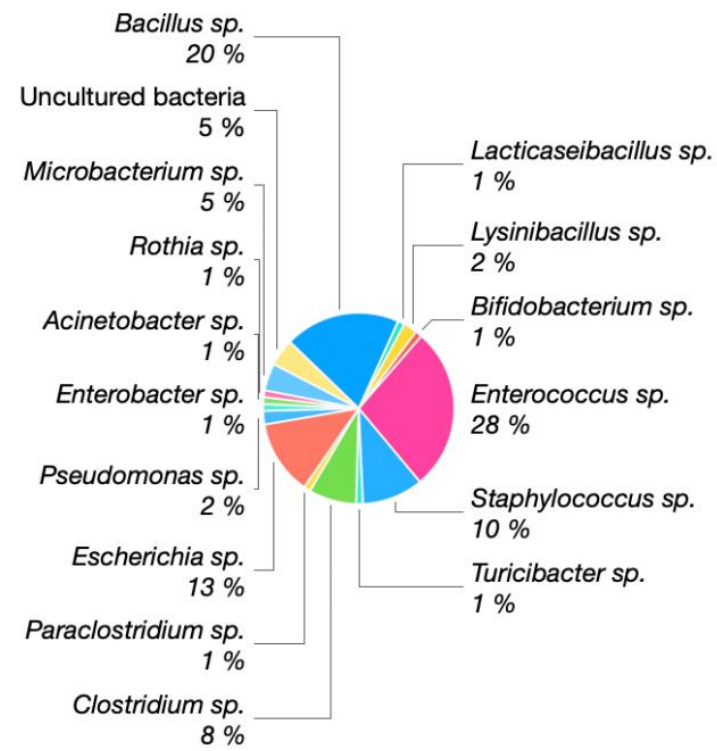

(a) Normo-weight microbiota specimens.

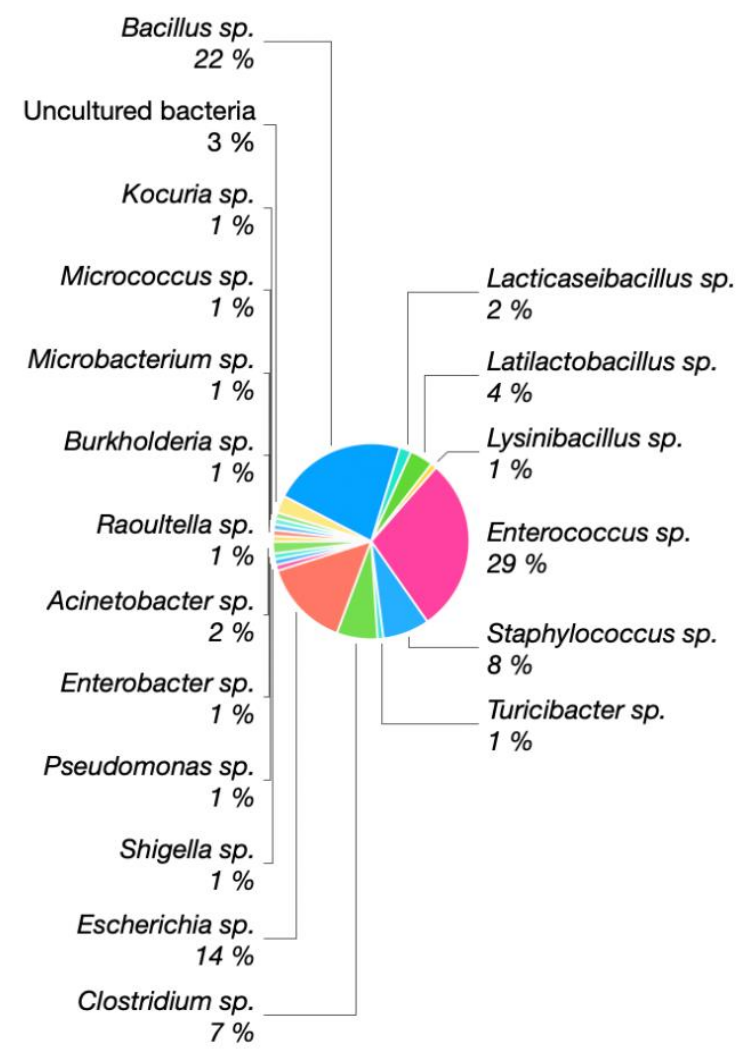

(b) Overweight and Obesity microbiota specimens.

Fig. 1 Relative abundance of genera in microbiota from 22 normo-weight children compared to 24 Overweight or Obesity treated with BPA. Numbers given in the pie chart correspond to this percentage.

Microbial community from the culturomics approach and the statistical analyses showed that the obese group had more diversity, richness (Chao1, Observe) and evenness (Shannon, InvSimpson) than the normo-weight group. As for other culturomics studies, these results lead to complement already adapted 
approaches by highlighting the bacteria that were considered "un-cultivable" as they might be playing an important role in the health balance and disease development [36].

Importantly, culturomics for isolating new bacterial species included toxicogenomics approach to describe novel organisms able to metabolize toxicants [6]. New bacterial species are subjected to a series of phenotypic, biochemical and genomic characterization (habitat, sporulation, shape, antibiotics profile, metabolism, fatty acids contents, genome sequencing/ assembly and annotation).

BPA Directed-Culturomics and Spore-forming microbiota taxa: Clostridium spp. y Bacillus spp.

We identify 43 spore-forming bacteria isolates from human gut microbiota with high BPA tolerance [>20 ppm]. They were isolated from general media, supplemented with BPA, in following order: GAMg (14 isolates), GAMa (18 isolates) and RCM (13 isolates) without any specific media for associated taxa. The overall mean values for colony counts were for GAMa + BPA 20 ppm 9X104 CFU/ml, GAMa + BPA 50

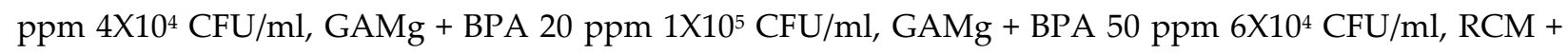
BPA $20 \mathrm{ppm}$ 9X104 CFU/ml, RCM + BPA $50 \mathrm{ppm} 5 \times 10^{4} \mathrm{CFU} / \mathrm{ml}$. The relative abundance of these sporeforming bacteria isolates, together with taxonomically closest species, maximum BPA concentration tolerated and specific media for isolation are detailed in Table 4 for normo-weight children specimens analyzed and Table 5 for specimens from children with obesity.

Table 4. BPA tolerant spore-forming bacteria taxa cultured from normo-weigth microbiota.

\begin{tabular}{|c|c|c|c|c|}
\hline $\begin{array}{c}\text { Representative } \\
\text { Isolates (\%) }\end{array}$ & $\begin{array}{l}\text { Closest species } \\
\text { Accession Number }\end{array}$ & $\begin{array}{c}\text { Similarity of } \\
\text { partial 16S } \\
\text { rRNA (\%) }\end{array}$ & $\begin{array}{l}\text { BPA [ppm] } \\
\text { Treatments }\end{array}$ & Media \\
\hline \multicolumn{5}{|c|}{ Firmicutes } \\
\hline 9 & Bacillus amyloliquefaciens |MZ359899.1| & 100 & 50 & $\mathrm{GAMa} / \mathrm{RCM}$ \\
\hline 4 & Bacillus vallismortis |KX462780.1| & 99.54 & 50 & RCM \\
\hline 4 & Clostridium disporicum |LC515630.1। & 99.53 & 50 & GAMg \\
\hline 22 & Clostridium paraputrificum |MN913836.1| & 100 & $20 / 50$ & $\mathrm{GAMa} / \mathrm{GAMg} / \mathrm{RCM}$ \\
\hline 4 & Clostridium perfringens |MT613499.1| & 99.75 & 20 & GAMg \\
\hline 9 & Paraclostridium benzoelyticum |AB973393.1| & 99.51 & $20 / 50$ & GAMg \\
\hline 39 & Paeniclostridium sordellii ICP014150.1। & 99.88 & $20 / 50$ & $\mathrm{GAMa} / \mathrm{GAMg} / \mathrm{RCM}$ \\
\hline 4 & Uncultured bacterium IGQ159075.1| & 97.30 & 20 & GAMa \\
\hline 4 & Uncultured bacterium |KF110610.1| & 99.88 & 50 & GAMa \\
\hline
\end{tabular}

All sequences were submitted to GenBank under the Accession Numbers: MZ612806-MZ612850. 
Table 5. BPA tolerant spore-forming bacteria taxa cultured from obese microbiota.

\begin{tabular}{|c|c|c|c|c|}
\hline $\begin{array}{c}\text { Representative } \\
\text { Isolates (\%) }\end{array}$ & $\begin{array}{l}\text { Closest species } \\
\text { Accession Number }\end{array}$ & $\begin{array}{c}\text { Similarity of } \\
\text { partial 16S } \\
\text { rRNA }(\%)\end{array}$ & $\begin{array}{l}\text { BPA [ppm] } \\
\text { Treatments }\end{array}$ & Media \\
\hline & Firmicutes & & & \\
\hline 14 & Bacillus amyloliquefaciens IKM853034.1| & 99.76 & $20 / 50$ & GAMa/GAMg \\
\hline 10 & Bacillus velezensis | MZ474622.1| & 99.88 & $20 / 50$ & GAMa \\
\hline 5 & Bacillus pumilus |HM055978.1| & 99.42 & 50 & GAMa \\
\hline 5 & Bacillus subtilis |KX950665.1| & 98.99 & 50 & GAMa \\
\hline 5 & Bacillus licheniformis IHQ290087.1| & 100 & 20 & $\mathrm{RCM}$ \\
\hline 5 & Bacillus paralicheniformis |MT645610.1| & 99.04 & 50 & GAMg \\
\hline 5 & Clostridium perfringens IMH69435.1 | & 99.65 & 50 & GAMa \\
\hline 24 & Clostridium paraputrificum IMN913836.1| & 99.88 & $20 / 50$ & GAMa/GAMg/RCM \\
\hline 5 & Clostridium symbiosum |LC515566.1| & 100 & 20 & $\mathrm{RCM}$ \\
\hline 5 & Clostridium tepidum |MF581527.1 | & 98.12 & 20 & GAMa \\
\hline 14 & Paraclostridium benzoelyticum IMT510437.1| & 99.88 & 50 & GAMa/GAMg \\
\hline 5 & Uncultured bacterium |HQ541237.1। & 99.12 & 20 & GAMg \\
\hline
\end{tabular}

* Pretreatment ethanol and bile acids. All sequences were submitted to GenBank under Accession Numbers: MZ612806-MZ612850.

In this catalogue of spore-forming isolates from normo-weight children, Clostridium spp. represented $30.44 \%$ and Bacillus spp. $13.05 \%$. In contrast, from obese children higher percentages were found, Clostridium spp. constituted $38.09 \%$ and Bacillus spp. $42.85 \%$. It is interesting to highlight that Paeniclostridium sordellii specimens with high BPA tolerance were isolated only from normo-weight children, where it is the most representative specie (39.13\%). If we focus on biodiversity at the species level, a total of 7 different species and 2 isolates categorized like Uncultured bacterium have been isolated from samples belonging to normal-weight children. In the case of the isolates belonging to children with obesity, a total of 11 species and an Uncultured bacterium have been cultured, highlighting the cultivation of 6 different species of the Bacillus genus compared to the 2 isolated from the samples of children with normo-weight.

\section{BPA biodegradation metabolic maps through WGS $^{\mathrm{T}}$ data mining}

The bioinformatics analysis carried out on the WGS of Type strains of closest species identified as cultivable species from microbiota showed a differential potential of BPA biodegradation and specific enzymes arsenal involved (Table 6). The genome mining allowed identifying specific clusters prone to degrade bisphenols. Bioinformatics tools and Pascal ad hoc programme allowed the exhaustive analysis 
of genomes making it a powerful prediction toxicomicrobiomics tool. According to the theoretical predictive results, overall microbiota naturally possessed an intermediate degree of BPA biodegradation potential by the different enzymatic pathways disclosed (BPA (I) $41 \%$, BPA (II) $36 \%$, BPA (III) $41 \%$, and BPA (IV) 39\%). Burkolderia, Bacillus, Raoultella, Acinetobacter, Micrococcus and Microbacterium species were clustered as biodegrader. The analysis showed that they harboured the more complete BPA biodegradation genetic clusters (> 50\%), while species of Bifidobacterium, Lactobacillus, Enterococcus, Clostridium, Paeniclostridium and Turicibacter did not contain representative percentages of the gene loci for BPA biodegradation encoding enzymes and were clustered as tolerant or resistant to BPA.

Table 6. Microbiota representative genera harboring gene loci for encoding differential BPA enzyme pathways

\begin{tabular}{|c|c|c|c|c|c|c|}
\hline BPA PATHWAYS DATA ANALYSIS & BPA (I) & BPA (II) & BPA (III) & BPA (IV) & Mean & BPA capacity \\
\hline Gut Representative Taxa (Genera) & $40 \%$ & $34 \%$ & $42 \%$ & $38 \%$ & $38 \%$ & Threshold* \\
\hline Acidaminococcus & $18 \%$ & $31 \%$ & $38 \%$ & $14 \%$ & $24 \%$ & Tolerant \\
\hline Acinetobacter & $52 \%$ & $52 \%$ & $55 \%$ & $51 \%$ & $52 \%$ & Biodegrader \\
\hline Actinomyces & $35 \%$ & $38 \%$ & $38 \%$ & $29 \%$ & $36 \%$ & Tolerant \\
\hline Akkermansia & $29 \%$ & $23 \%$ & $38 \%$ & $43 \%$ & $31 \%$ & Tolerant \\
\hline Anaerostipes & $24 \%$ & $31 \%$ & $38 \%$ & $14 \%$ & $27 \%$ & Tolerant \\
\hline Bacillus & $61 \%$ & $53 \%$ & $53 \%$ & $54 \%$ & $56 \%$ & Biodegrader \\
\hline Bifidobacterium & $24 \%$ & $23 \%$ & $38 \%$ & $29 \%$ & $27 \%$ & Tolerant \\
\hline Burkholderia & $76 \%$ & $62 \%$ & $75 \%$ & $71 \%$ & $71 \%$ & Biodegrader \\
\hline Clostridium & $13 \%$ & $17 \%$ & $22 \%$ & $14 \%$ & $16 \%$ & Resistant \\
\hline Desulfovibrio & $18 \%$ & $15 \%$ & $25 \%$ & $43 \%$ & $22 \%$ & Tolerant \\
\hline Eggerthella & $18 \%$ & $15 \%$ & $38 \%$ & $14 \%$ & $20 \%$ & Tolerant \\
\hline Enterococcus & $25 \%$ & $15 \%$ & $21 \%$ & $14 \%$ & $20 \%$ & Tolerant \\
\hline Escherichia & $56 \%$ & $23 \%$ & $38 \%$ & $43 \%$ & $41 \%$ & Biodegrader \\
\hline Flavonifractor & $29 \%$ & $31 \%$ & $38 \%$ & $14 \%$ & $29 \%$ & Tolerant \\
\hline Kocuria & $53 \%$ & $38 \%$ & $63 \%$ & $71 \%$ & $53 \%$ & Biodegrader \\
\hline Lactobacillus & $24 \%$ & $12 \%$ & $19 \%$ & $29 \%$ & $20 \%$ & Tolerant \\
\hline Lysinibacillus & $65 \%$ & $58 \%$ & $56 \%$ & $64 \%$ & $61 \%$ & Biodegrader \\
\hline Micrococcus & $53 \%$ & $46 \%$ & $50 \%$ & $71 \%$ & $53 \%$ & Biodegrader \\
\hline Microbacterium & $53 \%$ & $54 \%$ & $54 \%$ & $43 \%$ & $52 \%$ & Biodegrader \\
\hline Paraclostridium & $12 \%$ & $8 \%$ & $25 \%$ & $14 \%$ & $13 \%$ & Resistant \\
\hline Pseudomonas & $41 \%$ & $38 \%$ & $63 \%$ & $57 \%$ & $47 \%$ & Biodegrader \\
\hline Raoultella & $71 \%$ & $46 \%$ & $75 \%$ & $57 \%$ & $62 \%$ & Biodegrader \\
\hline Roseburia & $18 \%$ & $31 \%$ & $25 \%$ & $14 \%$ & $22 \%$ & Tolerant \\
\hline Rothia & $47 \%$ & $31 \%$ & $38 \%$ & $71 \%$ & $44 \%$ & Biodegrader \\
\hline Shigella & $41 \%$ & $8 \%$ & $38 \%$ & $43 \%$ & $31 \%$ & Tolerant \\
\hline Slackia & $29 \%$ & $31 \%$ & $50 \%$ & $43 \%$ & $36 \%$ & Tolerant \\
\hline Staphylococcus & $47 \%$ & $23 \%$ & $50 \%$ & $43 \%$ & $40 \%$ & Biodegrader \\
\hline Turicibacter & $12 \%$ & $0 \%$ & $25 \%$ & $14 \%$ & $11 \%$ & Resistant \\
\hline
\end{tabular}

*BPA Biodegrader $>39 \%-71 \%$; BPA Tolerant $>20 \%-38 \%$; BPA Resistant $<19 \%$; * Data analysis in Supplemental material

Genome mining data based on WGST representative BPA biodegradation analyses was achieved through the advances in next generation sequencing (NGS) and in silico tools allows performing an appropriate screening of genes of concern or interest in microbiota, such as biodegradation capacities or toxicomicrobiomics potential through bioinformatics, metagenomics or in silico analysis of cultivable 
isolates WGS [58, 59]. A better understanding of the microbiota ecology driven by the bioactive compounds, which are released by gut microbial components may drive towards better clinical interventions [60]. Genome mining done in the present study allowed BLAST driven searching for predicted BPA pathways. Pascal ad hoc programme analysed the type strain genomes making it a powerful prediction tool. Similarly, another useful prediction tool could be used as well as for BPA biodegradation pathways [61].

Interestingly, the species found exclusively in normo-weight children microbiota (Paraclostridium sp. and Bifidobacterium sp.) had a low BPA biodegradation potential, being clustered as BPA tolerant or resistant. However, the species from obese children (Kouria sp., Micrococcus sp., Burkolderia sp., Raoultella sp. and Shigella sp.) showed higher BPA degradation potential, being grouped as BPA biodegrader. Thus, a first trend of this analysis showed that microorganisms from obese children seemed to present more BPA biodegradation potential than normo-weight children.

Moreover, comparative data from wide metagenomics analysis regarding the variability of taxa composition in individuals with obesity and normo-weight, Firmicutes/Bacteroidetes (F/B) ratio constitutes a recognized biomarker for comparisons, as well as Actinobacteria and Proteobacteria relative abundances. F/B ratio showed higher values in obese than normo-weight individuals [62] as Actinobacteria appeared usually also higher in obese population. Conversely, Bacteroides and Proteobacteria were slightly higher in normo-weight populations [62]. In parallel, our BPA directedculturomics approach have demonstrated that Firmicutes was one of the more predominant populated taxa able to grow in BPA and showing biodegrader-like profiles (Bacillus, Staphylococcus, Micrococcus). However, we could not compare data from Bacteroidetes as no cultivable taxa were obtained through this approach. On the other hand, isolated Proteobacteria taxa able to grow from no-obese specimens were different and they harboured lower capacity of BPA biodegradation compared to those obtained in children with obesity, which compiled species with the highest percentage of BPA enzymatic gene loci (Escherichia coli, Escherichia fergusonii, Shigella flexneri, Pseudomonas parafulva, Enterobacter cancerogenus, Raoultella ornithinolytica, Acinetobacter radioresistens and Burkholderia contaminans). 
In this sense, it is important to consider the ecological role of those enzymes and their impact on the gut microbiota composition may have a huge influence on metabolizing and neutralizing BPA, by releasing metabolites that contribute to the modification of individual taxa microbial components on long-term basis [63].

Interestingly, specific transitory gut taxa identified with high potential of BPA biodegradation could be also used for environmental bioremediation purposes or plant probiotics. Several authors investigated the BPA removal capacity using bacterial strains from dessert soil that belong to Pseudomonas putida, Pseudomonas aeruginosa, Enterobacter cloacae, Klebsiella sp. and Pantoea sp. [64]. Degradation of BPA by Pseudomonas putida YC-AE1 was considered as a low cost effective and eco-friendly method compared to physical and chemical methods [65]. Similarly, a consortium isolated from river sediment (Terrimonas pekingensis and Pseudomonas sp.) was able to use BPS as the sole carbon source and was highly efficient to degrade $99 \%$ with an initial concentration of $50 \mathrm{mg} / \mathrm{L}$ in 10 days [66]. Gut bacteria harbouring laccases could be used for detoxification of several hazardous dietary contaminants and emerging ED through bioreactor with novel biocatalytic system based on active membranes and immobilized laccase technology [67].

\section{Conclusions}

We are exposed to obesogenic MDC, such as bisphenols and concretely to BPA. The pathophysiological impact of these obesogens seem to depend on inter-individual and diverse microbial gut composition, and we are just starting to understand how these microbiota consortia interact with host and how their enzymatic arsenals would shape those communities to build a functional human microbiome. Our results indicate that specific and differential gut enriched microbial isolates or consortia that resist, tolerate or biodegrade BPA were present in human-associated microbial communities and they harboured the specific gene encoding enzymes involved in biodegrading BPA and other obesogens, and that such enhancing enzymatic properties of the gut communities could perpetuate their modulation ecological actions, even after the exposure to obesogens or BPA should be present, impacting in health and disease host status. 


\section{Availability of supporting data}

Sequence files and metadata for all samples used in this study have been deposited in Genbank under the GenBank submission numbers: SUB10046802; SUB10052679. A full record of all raw analysis for culturomics and bioinformatics BPA-biodegradation prediction is included as Additional files.

\section{Availability of data and materials: Additional files and Special Files}

All data generated or analysed during the study are included in Additional files: Excel 1. Culturomics retrieving information; Excel 2. Culturing media and conditions; Excel 3. Bioinformatics for BPA Loci Prediction.

Special files are available under request to the Authors. Excel 4. Complete Bioinformatics for BPA Loci Prediction.

\section{Abbreviations}

MDC: Microbiota Disrupting Chemicals; BPA: Bisphenol A; ED: Endocrine Disruptors; NGP: NextGeneration Probiotics; MALDI-TOF MS: Matrix-Assisted Laser Desorption/Ionization Time Of Flight Mass Spectrometry; BPS: Bisphenol S; MeSH: Medical Subject Headings; BHI: Brain Heart Infusion; MRS: Man, Rogosa and Sharpe; RCM: Reinforced Clostridial Medium; GAMm: Gifu Anaerobic modified Medium; IPBLN: Institute of Parasitology and Biomedicine "López-Neyra"; NCBI: National Center of Biotechnology Information; WGS: Whole Genome Sequencing; BCB: Blood Culture Bottle; BRU: Brucella Medium; CBA: Columbia Blood Agar; CHRIS: Christensenella Medium; CNA: Columbia Naladixicacid Agar; COS: Columbia Agar Liquid Medium + 5\% Sheep Blood; CPVX: Chocolate agar + PolyViteX; GAM: Gifu Anaerobic Media; MB: Marine Broth; RM: R-Medium; RCA: Reinforced Clostridial Agar; SCM: Schaedler Medium; TSB: Trypticase Soy Broth; YCFA: Yeast Extract-Casein Hydrolysate-Fatty Acids; WC: Wilkins Chalgren; GMM: gut microbiota medium; LBS: Lactobacillus selection; Aer: Aerobic; AAn: Aerotolerant Anaerobe; SAn: Strictly Anaerobic; FAn: Facultative Anaerobe; CFU: Colony Forming Unit; GAMa: GAM agar; GAMg: GAM gelano; BbY: Bifidobacterium breve strain Yakult; LcS: Lactobacillus casei strain Shirota; NGS: Next Generation Sequencing. 


\section{Funding}

ALM has a Ph.D. contract through the EFSA Grant and the programme "Intensificación de la Investigación" University of Granada (2019-2020). PO has a contract "Garantía Juvenil" -FEDER-Junta de Andalucía. K. Cerk is under the EU-FORA Fellowship Programme. ALM, ARM, PO and MA. are part of the BIO-190 Research Group. They are also part of “UGR Plan Propio de Investigación 2019-2022”

\section{Authors' contributions}

MA conceptualized the rational of the Manuscript; ARM, PO and MU performed the core work of the culturomics literature review. ALM and ARM performed the experimental directed-culturing for the bacteria catalogue. ALM drafted and prepared the initial manuscript. JP performed the BPA bioinformatics and WGS data mining. All assessed the content of the manuscript and discussion and performed a critical comparison of full data. MA revised and commented on the final draft of the manuscript.

\section{Ethics approval and consent to participate}

Fecal sample library was obtained after corresponding approval of CEIC 20/12/2019.

\section{Consent for publication}

Not Applicable.

\section{Competing interests}

The authors declare that they have no competing interests.

\section{References}

1. Egusquiza RJ, Blumberg B. Environmental Obesogens and Their Impact on Susceptibility to Obesity: New Mechanisms and Chemicals. Endocrinology 2020, 161:bqaa024.

2. Aguilera M, Lamas B, Van Pamel E, Bhide M, Houdeau E, Rivas A. Editorial: Risk of Dietary Hazardous Substances and Impact on Human Microbiota: Possible Role in Several Dysbiosis Phenotypes. Frontiers in Microbiology. 2021, 12.

3. Cohen IC, Cohenour ER, Harnett KG, Schuh SM. BPA, BPAF and TMBPF Alter Adipogenesis and Fat Accumulation in Human Mesenchymal Stem Cells, with Implications for Obesity. Int J Mol Sci 2021, 22:5363.

4. Wang T, Li M, Chen B, Xu M, Xu Y, Huang Y, et al. Urinary bisphenol A (BPA) concentration associates with obesity and insulin resistance. J Clin Endocrinol Metab 2012, 97:E223-227.

5. Lai KP, Ng AH-M, Wan HT, Wong AY-M, Leung CC-T, Li R, et al. Dietary Exposure to the Environmental Chemical, PFOS on the Diversity of Gut Microbiota, Associated With the Development of Metabolic Syndrome. Front Microbiol 2018, 9:2552.

6. Rajkumar H, Mahmood N, Kumar M, Varikuti SR, Challa HR, Myakala SP. Effect of probiotic (VSL\#3) and omega-3 on lipid profile, insulin sensitivity, inflammatory markers, and gut colonization in overweight adults: a randomized, controlled trial. Mediators Inflamm 2014, 2014:348959. 
7. O'Toole PW, Marchesi JR, Hill C. Next-generation probiotics: the spectrum from probiotics to live biotherapeutics. Nat Microbiol 2017, 2:17057.

8. López-Moreno A, Acuña I, Torres-Sánchez A, Ruiz-Moreno Á, Cerk K, Rivas A, et al. Next Generation Probiotics for Neutralizing Obesogenic Effects: Taxa Culturing Searching Strategies. Nutrients 2021, 13:1617.

9. Lagier J-C, Armougom F, Million M, Hugon P, Pagnier I, Robert C, et al. Microbial culturomics: paradigm shift in the human gut microbiome study. Clinical Microbiology and Infection 2012, 18:1185-93.

10. Váradi L, Luo JL, Hibbs DE, Perry JD, Anderson RJ, Orenga S, et al. Methods for the detection and identification of pathogenic bacteria: past, present, and future. Chem Soc Rev 2017, 46:4818-32.

11. Lagier J-C, Hugon P, Khelaifia S, Fournier P-E, La Scola B, Raoult D. The Rebirth of Culture in Microbiology through the Example of Culturomics To Study Human Gut Microbiota. Clinical Microbiology Reviews 2015, 28:237-64.

12. Lagier J-C, Khelaifia S, Alou MT, Ndongo S, Dione N, Hugon P, et al. Culture of previously uncultured members of the human gut microbiota by culturomics. Nat Microbiol 2016, 1:1-8.

13. Aguilera M, Gálvez-Ontiveros Y, Rivas A. Endobolome, a New Concept for Determining the Influence of Microbiota Disrupting Chemicals (MDC) in Relation to Specific Endocrine Pathogenesis. Front Microbiol $2020,11$.

14. Louati I, Dammak M, Nasri R, Belbahri L, Nasri M, Abdelkafi S, et al. Biodegradation and detoxification of bisphenol A by bacteria isolated from desert soils. Biotech 2019, 9:228.

15. Joskow R, Barr DB, Barr JR, Calafat AM, Needham LL, Rubin C. Exposure to bisphenol A from bis-glycidyl dimethacrylate-based dental sealants. J Am Dent Assoc 2006, 137:353-62.

16. Gálvez-Ontiveros Y, Moscoso-Ruiz I, Rodrigo L, Aguilera M, Rivas A, Zafra-Gómez A. Presence of parabens and bisphenols in food commonly consumed in spain. Foods 2021, 10.

17. Stoker C, Andreoli MF, Kass L, Bosquiazzo VL, Rossetti MF, Canesini G, et al. Perinatal exposure to bisphenol A (BPA) impairs neuroendocrine mechanisms regulating food intake and kisspetin system in adult male rats. Evidences of metabolic disruptor hypothesis. Mol Cell Endocrinol 2020, 499:110614.

18. Chung YH, Han JH, Lee S-B, Lee Y-H. Inhalation Toxicity of Bisphenol A and Its Effect on Estrous Cycle, Spatial Learning, and Memory in Rats upon Whole-Body Exposure. Toxicol Res 2017, 33:165-71.

19. Hormann AM, Vom Saal FS, Nagel SC, Stahlhut RW, Moyer CL, Ellersieck MR, et al. Holding thermal receipt paper and eating food after using hand sanitizer results in high serum bioactive and urine total levels of bisphenol A (BPA). PLoS One 2014, 9:e110509.

20. Gao C-J, Kannan K. Phthalates, bisphenols, parabens, and triclocarban in feminine hygiene products from the United States and their implications for human exposure. Environment International 2020, 136:105465.

21. Vandenberg LN, Chahoud I, Heindel JJ, Padmanabhan V, Paumgartten FJR, Schoenfelder G. Urinary, circulating, and tissue biomonitoring studies indicate widespread exposure to bisphenol A. Cien Saude Colet 2012, 17:407-34.

22. Vandenberg LN, Hauser R, Marcus M, Olea N, Welshons WV. Human exposure to bisphenol A (BPA). Reproductive Toxicology 2007, 24:139-77.

23. Suyamud B, Thiravetyan P, Gadd GM, Panyapinyopol B, Inthorn D. Bisphenol A removal from a plastic industry wastewater by Dracaena sanderiana endophytic bacteria and Bacillus cereus NI. International Journal of Phytoremediation 2020, 22:167-75.

24. Vijayalakshmi V, Senthilkumar P, Mophin-Kani K, Sivamani S, Sivarajasekar N, Vasantharaj S. Biodegradation of Bisphenol A by Pseudomonas aeruginosa PAb1 isolated from effluent of thermal paper industry: Kinetic modeling and process optimization. Journal of Radiation Research and Applied Sciences. 2018, 11:56-65.

25. Thoene M, Dzika E, Gonkowski S, Wojtkiewicz J. Bisphenol S in Food Causes Hormonal and Obesogenic Effects Comparable to or Worse than Bisphenol A: A Literature Review. Nutrients 2020, 12:E532.

26. Wu L-H, Zhang X-M, Wang F, Gao C-J, Chen D, Palumbo JR, et al. Occurrence of bisphenol S in the environment and implications for human exposure: A short review. Sci Total Environ 2018, 615:87-98.

27. Li, G.; Zu, L.; Wong, P.-K.; Hui, X.; Lu, Y.; Xiong, J.; An, T. Biodegradation and detoxification of Bisphenol A with one newly-isolated strain Bacillus sp. GZB: Kinetics, mechanism and estrogenic transition. Bioresour Technol 2012, 114, 224-230.

28. Das, R.; Liang, Z.; Li, G.; Mai, B.; An, T. Genome sequence of a spore-laccase forming, BPA-degrading Bacillus sp. GZB isolated from an electronic-waste recycling site reveals insights into BPA degradation pathways. Arch Microbiol 2019, 201, 623-638. 

Gut Microbiota Harbor Versatile Molecular Pathways for Bisphenol A Biodegradation. International Journal of Molecular Sciences 2021, 22:4952.

30. López-Moreno A, Suárez A, Avanzi C, Monteoliva-Sánchez M, Aguilera M. Probiotic Strains and Intervention Total Doses for Modulating Obesity-Related Microbiota Dysbiosis: A Systematic Review and Metaanalysis. Nutrients 2020, 12:1921.

31. Tamaki H, Sekiguchi Y, Hanada S, Nakamura K, Nomura N, Matsumura M, et al. Comparative analysis of bacterial diversity in freshwater sediment of a shallow eutrophic lake by molecular and improved cultivation-based techniques. Appl Environ Microbiol 2005, 71:2162-9.

32. Yoon S-H, Ha S-M, Kwon S, Lim J, Kim Y, Seo H, et al. Introducing EzBioCloud: a taxonomically united database of 16S rRNA gene sequences and whole-genome assemblies. Int J Syst Evol Microbiol. 2017;67:1613-7.

33. Bilen M, Dufour J-C, Lagier J-C, Cadoret F, Daoud Z, Dubourg G, et al. The contribution of culturomics to the repertoire of isolated human bacterial and archaeal species. Microbiome 2018, 6:94.

34. Greub G. Culturomics: a new approach to study the human microbiome. Clinical Microbiology and Infection. 2012, 18:1157-9.

35. Locey KJ, Lennon JT. Scaling laws predict global microbial diversity. PNAS 2016, 113:5970-5.

36. Bilen M. Strategies and advancements in human microbiome description and the importance of culturomics. Microbial Pathogenesis 2020, 149:104460.

37. Diakite A, Dubourg G, Dione N, Afouda P, Bellali S, Ngom II, et al. Extensive culturomics of 8 healthy samples enhances metagenomics efficiency. PLOS ONE 2019, 14:e223543.

38. Park S-K, Kim M-S, Roh SW, Bae J-W. Blautia stercoris sp. nov., isolated from human faeces. International Journal of Systematic and Evolutionary Microbiology 2012, 62:776-9.

39. Pham T-P-T, Cadoret F, Alou MT, Brah S, Diallo BA, Diallo A, et al. 'Urmitella timonensis' gen. nov., sp. nov., 'Blautia marasmi' sp. nov., 'Lachnoclostridium pacaense' sp. nov., 'Bacillus marasmi' sp. nov. and 'Anaerotruncus rubiinfantis' sp. nov., isolated from stool samples of undernourished African children. New Microbes and New Infections 2017, 17:84-8.

40. Chang Y, Hou F, Pan Z, Huang Z, Han N, Bin L, et al. Optimization of Culturomics Strategy in Human Fecal Samples. Front Microbiol 2019, 0. doi:10.3389/fmicb.2019.02891.

41. Diakite A, Dubourg G, Dione N, Afouda P, Bellali S, Ngom II, et al. Optimization and standardization of the culturomics technique for human microbiome exploration. Sci Rep 2020, 10:9674.

42. Browne HP, Forster SC, Anonye BO, Kumar N, Neville BA, Stares MD, et al. Culturing of 'unculturable' human microbiota reveals novel taxa and extensive sporulation. Nature 2016, 533:543-6.

43. Durand GA, Pham T, Ndongo S, Traore SI, Dubourg G, Lagier J-C, et al. Blautia massiliensis sp. nov., isolated from a fresh human fecal sample and emended description of the genus Blautia. Anaerobe 2017, 43:47-55.

44. Traore SI, Azhar EI, Yasir M, Bibi F, Fournier P-E, Jiman-Fatani AA, et al. Description of 'Blautia phocaeensis' sp. nov. and 'Lachnoclostridium edouardi' sp. nov., isolated from healthy fresh stools of Saudi Arabia Bedouins by culturomics. New Microbes and New Infections 2017, 19:129-31.

45. Ghimire S, Wongkuna S, Kumar R, Nelson E, Christopher-Hennings J, Scaria J. Genome sequence and description of Blautia brookingsii SG772 sp. nov., a novel bacterial species isolated from human faeces. New Microbes and New Infections 2020, 34:100648.

46. Alou MT, Ndongo S, Frégère L, Labas N, Andrieu C, Richez M, et al. Taxonogenomic description of four new Clostridium species isolated from human gut: 'Clostridium amazonitimonense', 'Clostridium merdae', 'Clostridium massilidielmoense' and 'Clostridium nigeriense.' New Microbes and New Infections 2018, 21:128-39.

47. Yimagou EK, Tall ML, Baudoin JP, Raoult D, Bou Khalil JY. Clostridium transplantifaecale sp. nov., a new bacterium isolated from patient with recurrent Clostridium difficile infection. New Microbes and New Infections. 2019, 32:100598.

48. Tall ML, Lo CI, Yimagou EK, Ndongo S, Pham TPT, Raoult D, et al. Description of Clostridium cagae sp. nov., Clostridium rectalis sp. nov. and Hathewaya massiliensis sp. nov., new anaerobic bacteria isolated from human stool samples. New Microbes and New Infections 2020, 37:100719.

49. Gouba N, Yimagou EK, Hassani Y, Drancourt M, Fellag M, Mbogning Fonkou MD. Enterococcus burkinafasonensis sp. nov. isolated from human gut microbiota. New Microbes and New Infections 2020, 36:100702. 
50. Anani H, Guilhot E, Andrieu C, Fontanini A, Raoult D, Fournier PE. Prevotella ihumii sp. nov., a new bacterium isolated from a stool specimen of a healthy woman. New Microbes and New Infections 2019, 32:100607.

51. Bellali S, Naud S, Ndongo S, Lo CI, Anani H, Raoult D, et al. Corynebacterium pacaense sp. nov., Alistipes megaguti sp. nov., Alistipes provencensis sp. nov., 3 new bacteria isolated from fresh human stool specimens. New Microbes and New Infections 2019, 32:100593.

52. Hedberg ME, Israelsson A, Moore ERB, Svensson-Stadler L, Wai SN, Pietz G, et al. Prevotella jejuni sp. nov., isolated from the small intestine of a child with coeliac disease. International Journal of Systematic and Evolutionary Microbiology 2013, 63:4218-23.

53. Lagier J-C, Karkouri KE, Mishra AK, Robert C, Raoult D, Fournier P-E. Non contiguous-finished genome sequence and description of Enterobacter massiliensis sp. nov. Standards in Genomic Sciences 2013, 7:399.

54. Oishi K, Sato T, Yokoi W, Yoshida Y, Ito M, Sawada H. Effect of probiotics, Bifidobacterium breve and Lactobacillus casei, on bisphenol A exposure in rats. Biosci Biotechnol Biochem 2008, 72:1409-15.

55. Endo Y, Kimura N, Ikeda I, Fujimoto K, Kimoto H. Adsorption of bisphenol A by lactic acid bacteria, Lactococcus, strains. Appl Microbiol Biotechnol 2007, 74:202-7.

56. Wang Y, Rui M, Nie Y, Lu G. Influence of gastrointestinal tract on metabolism of bisphenol A as determined by in vitro simulated system. J Hazard Mater 2018, 355:111-8.

57. Fournier P-E, Drancourt M. New Microbes New Infections promotes modern prokaryotic taxonomy: a new section "TaxonoGenomics: new genomes of microorganisms in humans." New Microbes and New Infections 2015, 7:489.

58. Calatayud Arroyo M, García Barrera T, Callejón Leblic B, Arias Borrego A, Collado MC. A review of the impact of xenobiotics from dietary sources on infant health: Early life exposures and the role of the microbiota. Environmental Pollution 2021, 269:115994.

59. Abdelsalam NA, Ramadan AT, ElRakaiby MT, Aziz RK. Toxicomicrobiomics: The Human Microbiome vs. Pharmaceutical, Dietary, and Environmental Xenobiotics. Front Pharmacol 2020, 11:390.

60. Marchesi JR, Adams DH, Fava F, Hermes GDA, Hirschfield GM, Hold G, et al. The gut microbiota and host health: a new clinical frontier. Gut 2016, 65:330-9.

61. Rangwala SH, Kuznetsov A, Ananiev V, Asztalos A, Borodin E, Evgeniev V, et al. Accessing NCBI data using the NCBI Sequence Viewer and Genome Data Viewer (GDV). Genome Res 2020. doi:10.1101/gr.266932.120.

62. Riva A, Borgo F, Lassandro C, Verduci E, Morace G, Borghi E, et al. Pediatric obesity is associated with an altered gut microbiota and discordant shifts in Firmicutes populations. Environ Microbiol 2017, 19:95-105.

63. Ly LK, Doden HL, Ridlon JM. Gut feelings about bacterial steroid-17,20-desmolase. Molecular and Cellular Endocrinology 2021, 525:111174.

64. Louati I, Dammak M, Nasri R, Belbahri L, Nasri M, Abdelkafi S, et al. Biodegradation and detoxification of bisphenol A by bacteria isolated from desert soils. 3 Biotech 2019, 9:228.

65. Eltoukhy A, Jia Y, Nahurira R, Abo-Kadoum MA, Khokhar I, Wang J, et al. Biodegradation of endocrine disruptor Bisphenol A by Pseudomonas putida strain YC-AE1 isolated from polluted soil, Guangdong, China. BMC Microbiology 2020, 20:11.

66. Wang X, Chen J, Ji R, Liu Y, Su Y, Guo R. Degradation of Bisphenol S by a Bacterial Consortium Enriched from River Sediments. Bull Environ Contam Toxicol 2019, 103:630-5.

67. Barrios-Estrada C, Rostro-Alanis M de J, Parra AL, Belleville M-P, Sanchez-Marcano J, Iqbal HMN, et al. Potentialities of active membranes with immobilized laccase for Bisphenol A degradation. International Journal of Biological Macromolecules 2018, 108:837-44. 


\section{Supplementary Files}

This is a list of supplementary files associated with this preprint. Click to download.

- SuplementaryTableCulturomics1.xlsx 